\title{
Interpretação sísmica de reservatórios não-convencionais (shale gas) da região do Baixo de Miranga, porção central da Bacia do Recôncavo
}

\author{
Daniel Bono R. Vilas Boas*; Wilker Eduardo Souza, INCT-GP/IGEO/UFBA
}

Copyright 2018, SBGf - Sociedade Brasileira de Geofísica

Este texto foi preparado para a apresentação no VIII Simpósio Brasileiro de Geofísica, Salinópolis, 18 a 20 de setembro de 2018. Seu conteúdo foi revisado pelo Comitê Técnico do VIII SimBGf, mas não necessariamente representa a opinião da SBGf ou de seus associados. É proibida a reprodução total ou parcial deste material para propósitos comerciais sem prévia autorização da SBGf.

\section{Abstract}

The work is based on a seismostratigraphic interpretation of 3D and 2D data, as well as geophysical well logs, aiming the identification of regions with greater potential of nonconventional reservoirs, especially shale gas, in the central portion of the Recôncavo Basin. The interpretation used a sequence stratigraphy model applied to rifte basins, in order to identify moments of greater expansion of the lacustrine system, and thus to be considered as regions of higher hydrocarbon generation. Seismic attributes were then used in order to evaluate positive amplitude and negative frequency anomalies, which may be indicative of shale gas.

\section{Introdução}

A produção de hidrocarbonetos através do faturamento hidráulico diretamente na rocha geradora configura um processo com grande desenvolvimento ao longo do século $\mathrm{XXI}$. A rocha geradora portanto assume o caráter de reservatórios não-convencionais, sobretudo quando há alto conteúdo orgânico e uma susceptibilidade alta ao faturamento (especialmente na presença de falhas, fraturas e fissuras). Esses reservatórios presentes em rochas de folhelhos fraturados permitem a produção de óleo e gás, respectivamente sendo chamados de shale oil e shale gas.

A Bacia do Recôncavo é considerada uma bacia madura, com extenso histórico exploratório. Contudo, existem poucos estudos em relação ao potencial de reservatórios não-convencionais presentes na bacia, e este tipo de produção ainda não foi empregada em nenhuma das bacias sedimentares brasileiras, sendo, portanto uma barreira exploratória ainda a ser quebrada.

Em termos de viabilidade, a Bacia do Recôncavo foi escolhida para o estudo, por se tratar de uma bacia com comprovado potencial petrolífero, alta proximidade com grandes centros, facilitando a distribuição, relativamente apresenta menos embargos ambientais se comparados com outras regiões (como região Amazônica, ou região sudeste acima do Aquífero Guarani), e apresenta intervalos em que a rocha geradora se encontra em baixas profundidades.

O presente trabalho tem como principal objetivo a interpretação sísmica da região central da Bacia do Recôncavo. Para tanto, seguiu-se os princípios da estratigrafia de sequências aplicada a bacias rifte, inicialmente preconizada por Prosser (1993), e adaptada com os conceitos tectônicos de Gawthorpe \& Leeder (2000), desenvolvido no modelo de Holz et al. (2017).

O presente estudo está inserido no desenvolvimento do projeto de pesquisa GASBRAS - Apoio à Rede de Gás Não Convencional no Brasil (Convênio USP, UERJ, UFRGS, UFMG, UFBA); desenvolvido junto ao INCTGP/IGEO/UFBA.

\section{Área de estudo / Base de dados}

A área de estudo está compreendida na porção central da Bacia do Recôncavo (BA, Brasil), entre os lineamentos transcorrentes de Mata-Catu e Itanagra-Araçás, no chamado Baixo de Miranga. Os dados disponíveis ao estudo constam de um polígono sísmico pós-empilhado de aproximadamente $142 \mathrm{~km}^{2}$, dados de 19 poços e 25 linhas sísmicas pós-empilhadas (vide Figura 1), todos eles públicos cedido pela ANP.

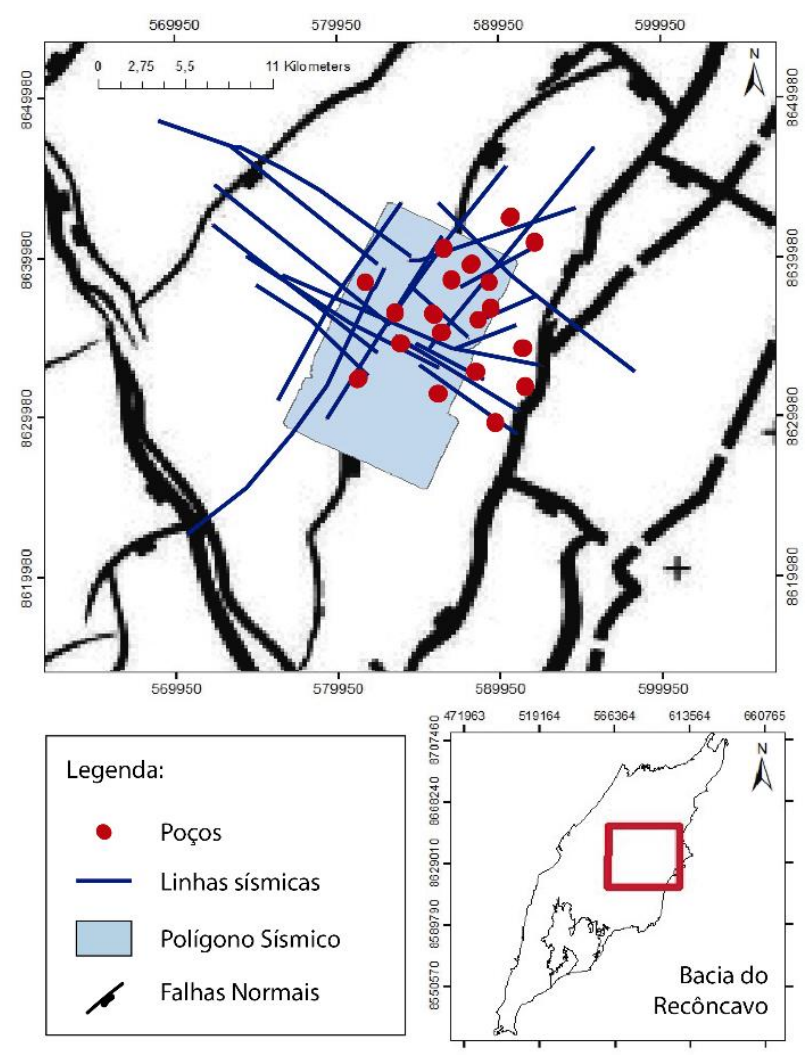

Figura 1 - Base de dados na região do Baixo de Miranga - porção central da Bacia do Recôncavo (BA, Brasil). 


\section{Método}

Os dados sísmicos e de poços foram todos analisados através do software de interpretação sísmica OpendTect, onde os poços foram amarrados às seções sísmicas através de confecção de sismogramas sintéticos. Também foram calculados diversos atributos sísmicos do traço complexo, entre eles: envelope, fase e frequência instantâneas, TecVA, similaridade, energia, sweetness, entre outros, aos quais foram utilizados tanto na análise sismoestratigráfica, quanto na posterior avaliação de anomalias indicadoras de potencial de reservatórios nãoconvencionais.

Ao longo da interpretação sísmica foi utilizado o modelo de estratigrafia de sequências para bacias do tipo rifte de Holz et al. (2017), onde a tectônica assume o principal papel de agente criador de espaço de acomodação. Tal aspecto, caracteriza os chamados tratos de sistemas tectônicos, onde foram avaliados dentro das sequências deposicionais de terceira ordem da fase sin-rifte, os tratos tectônicos de desenvolvimento (TTDR) e final de rifte (TTFR). O primeiro sendo caracterizado por expansão do sistema lacustre e característica geral retrogradacional a agradacional enquanto que o segundo (TTFR) apresenta aspecto progradacional, com aumento dos sistemas flúviodeltaicos frente ao lacustre. Estes tratos tem como limite entre eles a superfície de máximo rifteamento (SMR), ao qual representa o momento de maior expansão do ambiente lacustre, e assim, maior argilosidade (interpretado como maiores valores de raios-gama nos perfis de poços) e se apresenta como uma superfície de downlaps ao longo das seções sísmicas.

\section{Interpretação sísmica}

A análise estratigráfica iniciou com a confecção de seções de correlação de poços (vide figuras $3 C$ e 3D), seguindo pela interpretação das linhas sísmicas e dos dados sísmicos 3D, como pode ser visto em uma inline presente nas figuras $3 \mathrm{~A}$ e $3 \mathrm{~B}$.

Basicamente, foram identificadas três sequências deposicionais de terceira ordem ao longo da fase rifte, chamadas de sequências Rifte 1, Rifte 2 e Rifte 3, limitadas inferiormente pelas discordâncias DR1, DR2 e DR3, respectivamente. Dentro das duas primeiras sequências foram identificadas na análise dos poços pela mudança do padrão retrogradacional para progradacional (como pode ser visto nos perfis de raios gama dos poços presentes na Figura $3 C$ ), marcadas respectivamente pelas superfícies de máximo rifteamento SMR1 e SMR2. Estas também foram identificadas como superfícies de downlap nas seções do polígono sísmico, como é ilustrado na Fig. 3B. O resumo da interpretação sismoestratigráfica é apresentada em comparação com a carta cronoestratigráfica da Bacia do Recôncavo de Silva et al. (2007), ilustrada na Figura 2.

Sendo assim, os intervalos de maior potencial de geração de hidrocarbonetos foram associados aos momentos de maior expansão do sistema lacustre, intervalos de desenvolvimento de rifte: TTDR1 e TTDR2, por apresentar ambiente possivelmente mais redutor, e assim maior preservação de matéria orgânica, bem como maior espessura sedimentar de modo a facilitar as condições de maturação da matéria orgânica, e assim maior geração de hidrocarbonetos. Os intervalos descritos são destacados nas figuras $3 \mathrm{~B}$ e $3 \mathrm{D}$.

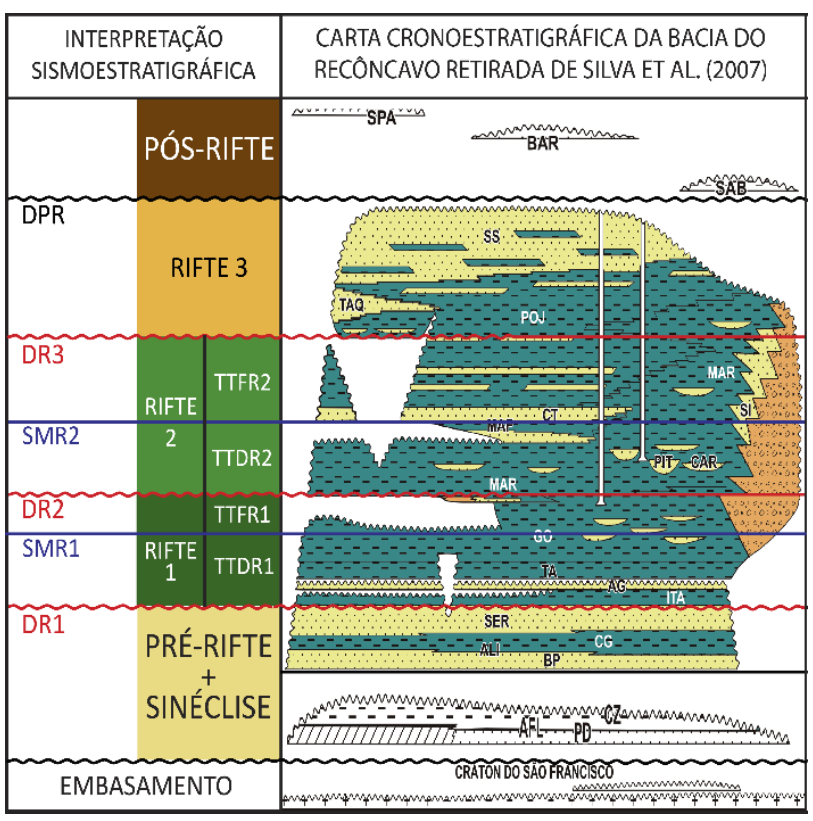

Figura 2 - Interpretação estratigráfica sobre o modelo cronoestratigráfico da Bacia do Recôncavo, extraído de Silva et al. (2007).

\section{Análise de atributos}

Após a análise estratigráfica, e assim definido os intervalos de maior potencial de geração de hidrocarbonetos (TTDR1 e TTDR2), a interpretação seguiu para a análise de atributos sísmicos do traço complexo, especialmente dentro do intervalo dessas regiões.

Foram utilizados diversos atributos sísmicos como amplitude instantânea (envelope), fase e frequência média instantânea, TecVA (Técnica Volume de Amplitudes, ou pseudorelief), similaridade (medida da coerência) e o atributo denominado sweetness (Hart, 2008). Este pode ser calculado como:

$$
S=E / \sqrt{f}
$$

Sendo $E$ o envelope (amplitude instantânea) e $f$ a frequência média instantânea.

Comparando-se os atributos de envelope, frequência média instantânea e sweetness com o intervalo de maior potencial de geração interpretado na inline 142 (Figura 4), é possível identificar anomalias positivas de sweetness dentro do intervalo de $600 \mathrm{~ms}$ e $900 \mathrm{~ms}$ (Figura 4C), confinado no intervalo do desenvolvimento do Rifte 2 (TTDR2). Tal anomalia sugere alta presença de gás, e comparando-se com as leituras de raios gama e de amostras de calha nos perfis de poços, é possível 
identificar que parte dessas anomalias são associadas a rochas como folhelhos lacustres escuros, de ambientes redutores. Além, disso foram analisados dados de perfis geoquímicos, onde ao longo do poço 3MGP35D (amarrado à seção da Figura 4D), foram identificados valores de carbono orgânico total (COT) superior à $3 \%$, inclusive dentro do intervalo indicado, o que sugere a possibilidade da geração ao longo desses depósitos.

\section{Discussão e Conclusões}

A interpretação sismoestratigráfica identificou três sequências deposicionais de terceira ordem dentro da fase rifte, e internas às duas primeiras sequências, dois intervalos de desenvolvimento de sistema lacustre, o trato tectônico de desenvolvimento do rifte 1 (TTDR1) e do rifte 2 (TTDR2). Estes, foram então considerados as regiões mais propensas à geração de hidrocarbonetos, se valendo das melhores condições de espaço de acomodação sedimentar e ambiente redutor, permitindo assim maior preservação de matéria orgânica, além de pacote sedimentar sotoposto de modo a favorecer a entrada dos depósitos nas janelas de gás e de óleo.

Em seguida, as anomalias de sweetness identificadas ao longo do intervalo do TTDR2, sugerem a possibilidade de folhelhos com bom potencial de geração (inclusive observado em valores de COT) que podem apresentar maior conteúdo de gás, o que resulta tanto em alto contraste de impedância acústica com os depósitos vizinhos (alta amplitude instantânea) e comumente mais baixas frequências. A região das anomalias sugerem portanto, os principais alvos para a presença de reservatórios não-convencionais da região, especialmente relacionados à shale gas.

\section{Agradecimentos}

Os autores agradecem ao INCT-GP/IGEO/UFBA por todos os recursos materiais, de infraestrutura e científicos fornecidos para a execução do estudo, ao FINEP pelo financiamento do projeto GASBRAS, ao qual se insere o presente trabalho, e ao CNPq e FAPESB pelas bolsas de pesquisa.

\section{Referências}

Gawthorpe, R. L., \& Leeder, M. R. (2000). Tectonosedimentary evolution of active extensional basins. Basin Research, 12(3-4), 195-218.

Hart, B. S. (2008). Channel detection in 3-D seismic data using sweetness. AAPG bulletin, 92(6), 733-742.

Holz, M., Vilas-Boas, D. B., Troccoli, E. B., Santana, V. C., \& Vidigal-Souza, P. A. (2017). Conceptual Models for Sequence Stratigraphy of Continental Rift Successions. In Stratigraphy \& Timescales (Vol. 2, pp. 119-186). Academic Press.

Prosser, S. (1993). Rift-related linked depositional systems and their seismic expression. Geological Society, London, Special Publications, 71(1), 35-66.

Silva, O. D., Caixeta, J. M., Milhomem, P. D. S., \& Kosin, M. D. (2007). Bacia do Recôncavo. Boletim de Geociências da PETROBRAS, 15(2), 423-431. 

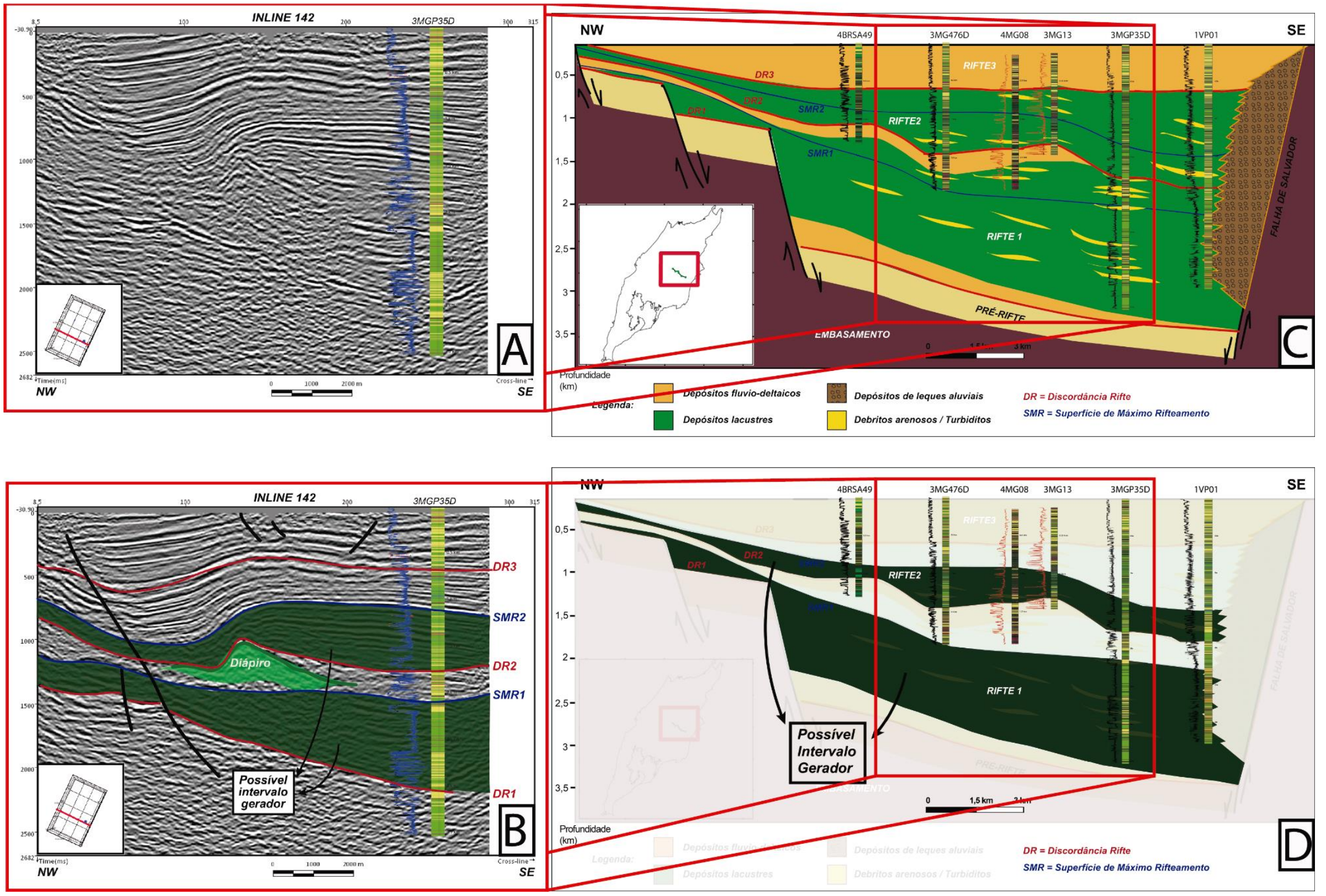

Figura 3 - Interpretação sísmica da Inline 142 do polígono sísmico: (A) seção não-interpretada, (B) seção interpretada; em comparação com a seção geológica de correlação de poços: (C) com a interpretação estratigráfica, (D) com o destaque do intervalo de maior potencial de rocha geradora. 

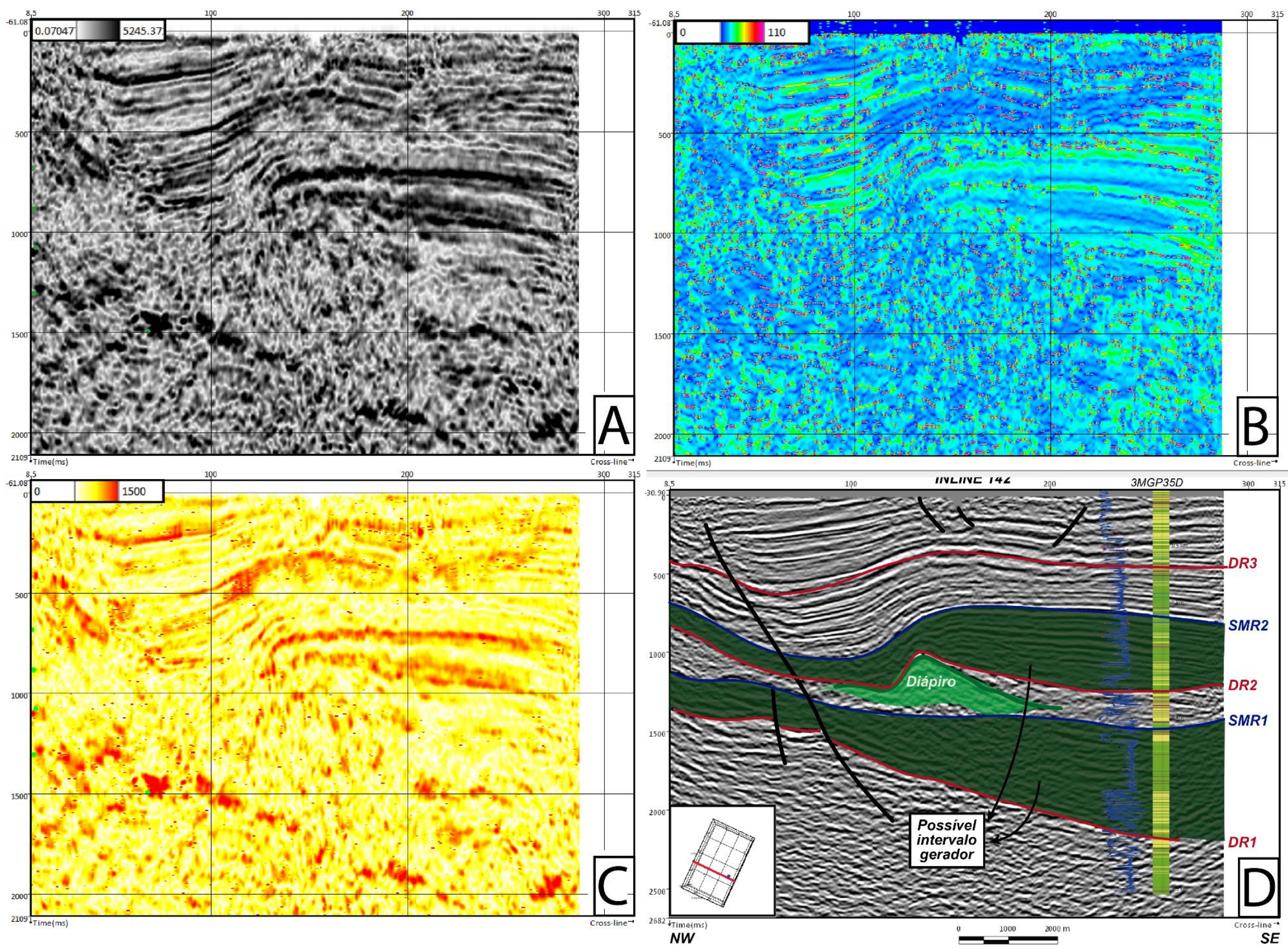

Figura 4 - Inline 142 com o atributo amplitude instantânea (A), frequência média instantânea (B), sweetness (C), e em amplitude sobreposta à interpretação sismoestratigráfica $(D)$. 